\title{
BAND GAP ENERGY OF CHALCOPYRITE THIN FILM SOLAR CELL ABSORBERS DETERMINED BY SOFT X-RAY EMISSION AND ABSORPTION SPECTROSCOPY
}

\author{
M. Bär, L. Weinhardt, S. Pookpanratana, and C. Heske \\ Department of Chemistry, University of Nevada, Las Vegas (UNLV), 4505 Maryland Pkwy., Las \\ Vegas, NV 89154-4003, USA \\ S. Nishiwaki and W.N. Shafarman \\ Institute of Energy Conversion (IEC), University of Delaware, Newark, 451 Wyoming Rd., Newark, \\ DE 19716, USA \\ O. Fuchs and M. Blum \\ Experimentelle Physik II, Universität Würzburg, Am Hubland, D-97074 Würzburg, Germany \\ W. Yang and J.D. Denlinger \\ Advanced Light Source (ALS), Lawrence Berkeley National Laboratory, 1 Cyclotron Rd, Berkeley, \\ CA 94720, USA
}

\begin{abstract}
The chemical and electronic structure of highefficiency chalcopyrite thin film solar cell absorbers significantly differs between the surface and the bulk. While it is widely accepted that the absorber surface exhibits a Cu-poor surface phase with increased band gap $\left(E_{g}\right)$, a direct access to the crucial information of the depth-dependency of $E_{g}$ is still missing. In this paper, we demonstrate that a combination of x-ray emission and absorption spectroscopy allows a determination of $E_{g}$ in the surface-near bulk and thus complements the established surface- and bulk-sensitive techniques of $E_{g}$ determination. As an example, we discuss the determination of $\mathrm{E}_{\mathrm{g}}$ for a $\mathrm{Cu}(\mathrm{In}, \mathrm{Ga}) \mathrm{Se}_{2}$ absorber [(1.52 \pm 0.20) eV].
\end{abstract}

\section{INTRODUCTION}

Thin film solar cells based on
$\mathrm{Cu}\left(\ln _{1-x} \mathrm{Gax}\right)\left(\mathrm{S}_{\mathrm{YSe}} \mathrm{Se}_{1-\mathrm{Y})_{2}}\right.$ ("CIGSSe") chalcopyrite absorbers are promising candidates to supersede the current state-of-the-art Si wafer-based photovoltaics. CIGSSe solar cells have already reached efficiencies close to $20 \%$ on a laboratory scale [1] and $13.4 \%$ on large areas (3459 $\mathrm{cm}^{2}$ [2]). For chalcopyrite absorbers in high-efficiency cells, the surface composition significantly deviates from the stoichiometric $\mathrm{Cu}$ : $(\mathrm{In}+\mathrm{Ga}):(\mathrm{S}+\mathrm{Se})=1: 1: 2$ bulk composition. Surfacesensitive characterization techniques generally indicate a Cu-poor surface composition [3-7]. In consequence, the formation of an ordered defect compound at the absorber surface was suggested [8,9]. Defect chalcopyrites with a composition ranging from $1: 2: 3.5$
$[8,10]$ over $1: 3: 5$ [11-14], $1: 5: 8[14,15], 1: 7: 11$ [16] to $1: 11: 17$ [16] were proposed, and corresponding (bulk) samples were synthesized. Optical characterization of these bulk materials shows that the defect (Cu-poor) chalcopyrites have larger band gap energies $\left(E_{g}\right)$ compared to their stoichiometric counterparts [10-13, 14-16]. For solar cell absorbers, direct determination of the surface band gap by UV photoelectron spectroscopy (UPS) and inverse photoemission (IPES) showed that $E_{g}$ is indeed widened at the Cu-poor surface of absorbers with stoichiometric bulk composition [4-7].

To date, the thickness and extent of the region with widened band gap is not known. No measurement technique so far established in the chalcopyrite community is able to directly probe $E_{g}$ in the surfacenear bulk and thus form the bridge between very surface-sensitive UPS and IPES measurements (i.e., the electronic surface band gap) and bulk-sensitive optical measurements (i.e., the optical bulk band gap). Note that band gap profiles based on composition gradients determined by (destructive) depth profiling techniques cannot play this role, since they do not consider the influence of $\mathrm{Cu}$ depletion on $\mathrm{E}_{\mathrm{g}}$ [17].

While x-ray emission spectroscopy (XES) probes occupied electronic states, x-ray absorption spectroscopy (XAS) probes unoccupied states. Thus, by combining these two techniques, it is possible to probe $E_{g}$ (in the presence of a core hole, i.e. not in the ground state). 


\section{EXPERIMENTAL}

This investigation is based on a S-free $\mathrm{Cu}\left(\operatorname{In}_{1-x} \mathrm{Gax}_{\mathrm{x}}\right) \mathrm{Se}_{2}$ "CIGSe"/Mo/glass test structure. The chalcopyrite absorber film (approx. $2 \mu \mathrm{m}$ thick) was prepared using multi-source thermal co-evaporation without intentional composition gradients. The Mocoated soda-lime glass substrate was held at $550^{\circ} \mathrm{C}$ during absorber formation (see Ref. 18 for more details). In order to minimize sample contamination due to air exposure, the samples were individually packed in dry nitrogen immediately after preparation. The sample exposure time to ambient air (before the first characterization) could thus be limited to less than 5 $\min$.

XES/XAS spectra were collected in the SXF endstation of Beamline 8.0 at the Advanced Light Source (ALS), Lawrence Berkeley National Laboratory (endstation base pressure below $5 \cdot 10^{-8}$ mbar). The XAS spectra were recorded in the total fluorescence yield mode using a channeltron mounted in front of the sample, and the XES spectra were taken with the permanently installed SXF spectrometer.

\section{RESULTS AND DISCUSSION}

Fig. 1 shows the $\mathrm{Cu}_{3}$ XES and XAS spectra of the investigated CIGSe sample. For comparison, spectra of metallic $\mathrm{Cu}$ are also shown. Since XES and XAS probe occupied and unoccupied states, respectively, the combination of both spectra gives information about $E_{g}$. Note that the band gap that can be determined from these measurements represents the band gap in the presence of a core hole (in our case a Cu $2 p_{3 / 2}$ core hole). This band gap " $E_{g}$ " is an approximation for the ground state band gap due to the potential existence of core excitonic features in the XAS spectra. If a core exciton is present, the XAS onset is observed at lower photon energies, and hence " $E_{g}$ " represents a lower limit to the ground state band gap.

" $E_{g}$ " was determined as the intersection of the baseline with a linear extrapolation of the leading XES and XAS edges. For the CIGSe absorber an " $E_{g}$ " of [1.52 \pm 0.20$] \mathrm{eV}$ is found, while the Cu metal reference shows no "gap" between the respective XES and XAS spectra (as expected).

The derived CIGSe " $\mathrm{E}_{\mathrm{g}}$ " value is considerably bigger than the bulk band gap: according to its bulk composition of $X=0.21$, as determined by energy dispersive $\mathrm{X}$-ray analysis, the bulk band gap energy was approximated [17] to $1.12 \mathrm{eV}$. Since the core-hole influence turns the derived " $E_{g}$ " values into a lower-

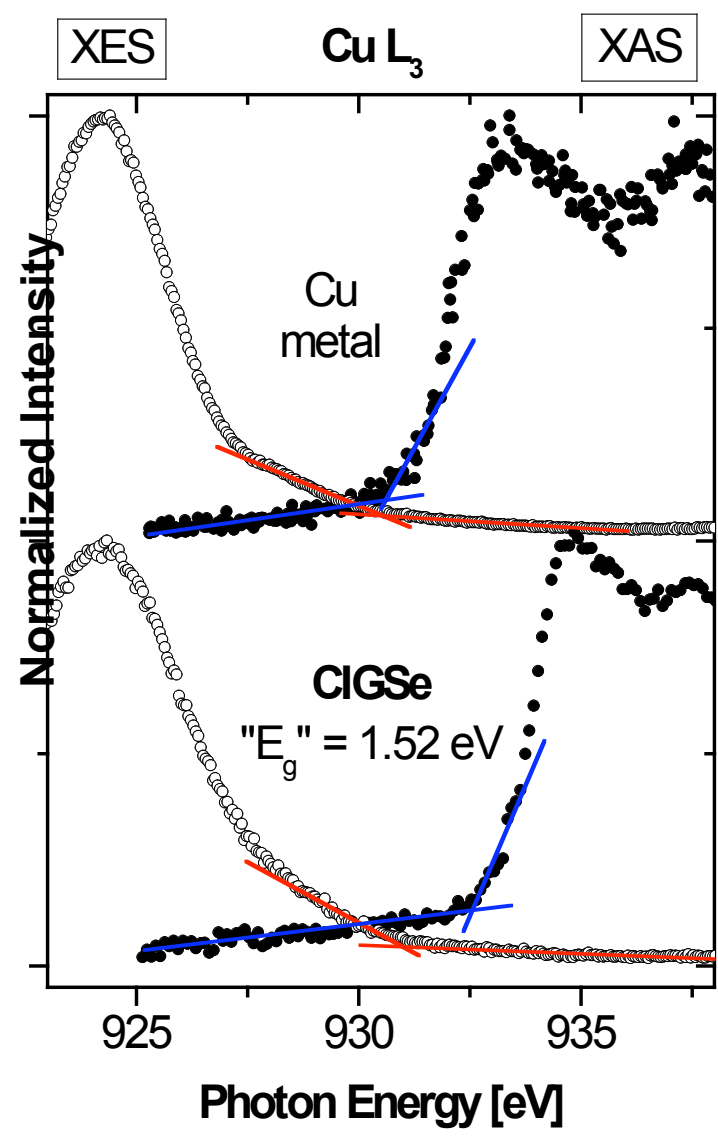

Fig. 1. $C u L_{3} x$-ray emission (XES, left) and absorption (XAS, right) spectra of the investigated CIGSe (bottom spectra) sample. For comparison, the spectra of metallic $\mathrm{Cu}$ (top spectra) are also shown. The linear approximation of the leading edges for the determination of " $E_{g}$ " (a lower bound for the true band gap value - see text) is indicated by the solid blue and red lines. The given band gap energy " $E_{g}$ " has an error of $\pm 0.20 \mathrm{eV}$.

bound approximation for the ground state band gap, the observation of an increased " $E_{g}$ " value unambiguously indicates an increased ground state band gap as well.

In order to understand the observed difference, the information depth of the XES and XAS measurements has to be quantified. Since the photons involved in XAS (in total fluorescence yield mode) have similar attenuation lengths as those in XES, we limit our respective considerations to the latter:

The intensity of the emission from a material $A$ of limited thickness $I^{A}(x)$ can be written as

$$
\mathrm{I}^{\mathrm{A}}(\mathrm{x})=\mathrm{I}_{\mathrm{ref}}^{\mathrm{A}}\left(1-\mathrm{e}^{-\frac{\mathrm{x}}{\lambda^{*}}}\right)
$$


where $\mathrm{I}_{\text {ref }}^{\mathrm{A}}$ denotes the reference emission intensity of material A with infinite thickness ("infinite" corresponds to a material thickness that results in a saturated emission intensity). Furthermore, we define the effective attenuation length $\lambda^{*}$ by

$$
\frac{1}{\lambda *}=\left(\frac{1}{\lambda_{\mathrm{exc}} \cdot \sin \alpha}\right)+\left(\frac{1}{\lambda_{\mathrm{em}} \cdot \sin \beta}\right)
$$

where $\lambda_{\text {exc }}$ and $\lambda_{\text {em }}$ are the attenuation lengths in material A for the excitation and emission energy, respectively. $\alpha$ and $\beta$ are the angles of excitation and emission relative to the sample surface, respectively (in our case $\alpha=\beta=45^{\circ}$ ). This effective attenuation length takes into account that, for an XES or XAS event to be recorded, a photon-in-photon-out process needs to take place.

Fig. 2 shows the attenuation length $\lambda$ (from Ref. [19]) as a function of photon energy for a CIGSe sample (with $X=0.21$ and thus a density of $5.73 \mathrm{~g} / \mathrm{cm}^{3}$ [17] and $\left.\alpha=\beta=45^{\circ}\right) \cdot \lambda$ ranges from approx. $20 \mathrm{~nm}$ for low photon energies to 200-300 $\mathrm{nm}$ for higher photon energies. At approx. 450, 670, and $930 \mathrm{eV}$, pronounced features can be observed, which can be ascribed to In $M_{4,5}$, In $M_{2,3}$, and $C u L_{2,3}$ absorption edges. Since we use the $\mathrm{Cu}_{3}$ emission and absorption to determine "Eg", $\lambda_{\text {exc }}$ (at $1000 \mathrm{eV}$ ) and $\lambda_{\text {em }}($ at $925 \mathrm{eV}$ ) are 242 and $287 \mathrm{~nm}$, respectively. Using Eq. (2), $\lambda^{*}$ can then be calculated to $93 \mathrm{~nm}$. Assuming that the information depth is defined as the thickness from which $90 \%$ of the overall signal is collected, we can use Eq. (1) to determine an approximate information depth of $213 \mathrm{~nm}$. Note that this is an approximate value, since the specific morphology of the surface is not taken into account. As is well known, corrugations of CIGSe surfaces can easily amount to several tens of nanometers.

The observed difference between the " $E_{g}$ " value determined from our XES and XAS measurements [(1.52 \pm 0.20$) \mathrm{eV}]$ and the $E_{g}$ based on the bulk composition $(1.12 \mathrm{eV})$ can thus be discussed on the basis of different information depths. The fact that the band gap widening is detected with XES and XAS (i.e., with an information depth of $\sim 213 \mathrm{~nm}$ ) indicates that the Cu-poor, band gap-widened surface phase must be significantly extended into the bulk of the investigated CIGSe absorber.

Comparing the derived information depth of $213 \mathrm{~nm}$ with the information depths (a few $\mu$ m or a few $\mathrm{nm}$ ) of established techniques of $E_{g}$ determination (optical spectroscopy and UPS/IPES, respectively), it is now possible to derive band gap information from the

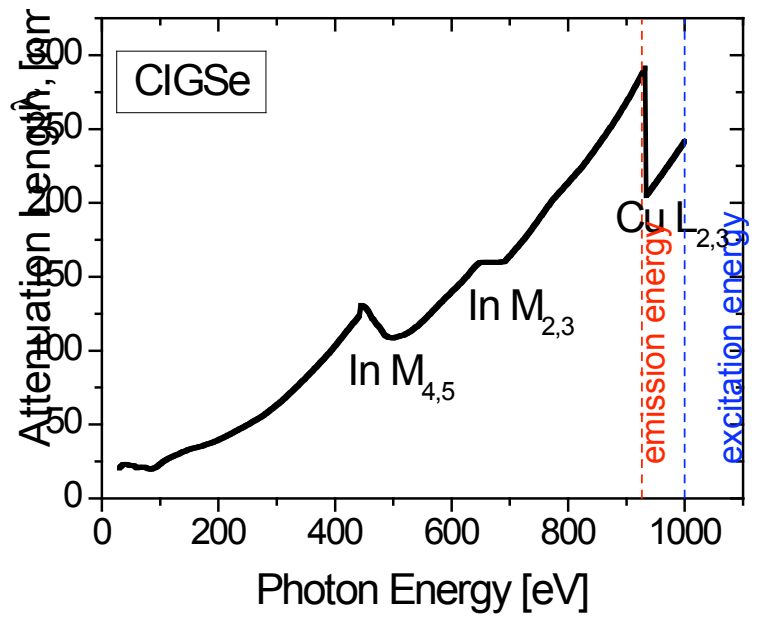

Fig. 2. Attenuation length (from Ref. [19]) as a function of photon energy, computed for the bulk composition of the investigated CIGSe sample.

intermediate depth regime between optical bulk band gaps and electronic surface band gaps. Note that, by using different absorption edges (if available) for the " $E_{g}$ " determination by XES/XAS, it is even possible to vary the XES/XAS information depth in discrete steps, provided the probed element has a significant contribution to both, the valence band maximum and conduction band minimum (as in the present case of $\mathrm{Cu})$.

In the near future, we are planning to extend our experiments to absorbers with different $X$ - and $Y$ composition, including wide-gap chalcopyrites such as CulnS $\mathrm{S}_{2}$ and $\mathrm{CuGaSe}_{2}$, in order to systematically investigate the depth-dependence of $E_{g}$ as a function of composition.

\section{SUMMARY \& CONCLUSION}

We have shown that the combination of $x$-ray emission (XES) and $x$-ray absorption (XAS) spectroscopy is uniquely suited to gain direct access to electronic band gaps in the surface-near bulk region (in the present case with an information depth of approx. $213 \mathrm{~nm}$ ). As an example, we have determined the surface-near electronic band gap for a $\mathrm{Cu}(\mathrm{In}, \mathrm{Ga}) \mathrm{Se}_{2}$ absorber to be $(1.52 \pm 0.20) \mathrm{eV}$. The combination of XES and XAS thus closes the "information gap" between established surface- and bulk-sensitive techniques to determine the band gap, opening a new route for the depth-dependent investigation of the electronic structure of chalcopyrite thin film solar cell absorbers. 


\section{Acknowledgement}

We acknowledge funding by the National Renewable Energy Laboratory under subcontract XXL-5-44205-12 and ADJ-1-30630-12, and by the U.S. DoE at the ALS under Contract No. DE-AC02-05CH11231. M.B. is grateful for sponsorship by the Deutsche Forschungsgemeinschaft (DFG) within the EmmyNoether-Programm.

\section{References}

[1] I. Repins et al., Prog. Photovolt. 16, 235 (2008).

[2] Y. Tanaka et al., Proc. $17^{\text {th }}$ EU-PVSEC, Munich, Germany (2001), 989.

[3] D. Schmid et al., Appl. Surf. Sci. 103, 409 (1996).

[4] M. Morkel et al., Appl. Phys. Lett. 79, 4482 (2001).

[5] L. Weinhardt et al., Thin Solid Films 431-432, 272 (2003).
[6] A. Meeder, et al., J. Phys. Chem. Sol. 64, 1553 (2003).

[7] L. Weinhardt et al., Appl. Phys. Lett. 86, 062109 (2005).

[8] J.R. Tuttle et al., Solar Cells 30, 21 (1991).

[9] D. Schmid et al., J. Appl. Phys. 73, 2902 (1993).

[10] T. Tanaka et al., J. Appl. Phys. 81, 7619 (1997).

[11] H.Z. Xiao et al., J. Appl. Phys. 76, 1503 (1994).

[12] S. Nishiwaki et al., Mat. Res. Soc. Symp. Vol. 763, B.5.18 (2003).

[13] S. Lehmann et al., Thin Solid Films 511-512, 623 (2006).

[14] M León et al., Phys. Stat. Sol. (a) 203, 2913 (2006).

[15] L. Durán et al., J. Phys. Chem. Sol. 64, 1907 (2003).

[16] I.V. Bodnar et al., Semiconductors 38, 197 (2004).

[17] M. Bär et al., J. Appl. Phys. 96, 3857 (2004).

[18] M. Gossla and W.N. Shafarman, Thin Solid Films 480-481, 33 (2005).

[19] B.L. Henke et al., CXRO database for x-ray attenuation length, http://henke.lbl.gov/optical 